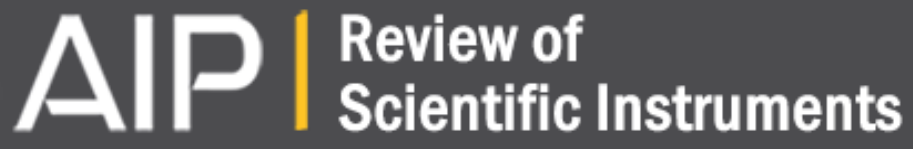

\section{A simple electron time-of-flight spectrometer for ultrafast vacuum ultraviolet photoelectron spectroscopy of liquid solutions}

C. A. Arrell, J. Ojeda, M. Sabbar, W. A. Okell, T. Witting, T. Siegel, Z. Diveki, S. Hutchinson, L. Gallmann, U. Keller, F. van Mourik, R. T. Chapman, C. Cacho, N. Rodrigues, I. C.E. Turcu, J. W.G. Tisch, E. Springate, J. P. Marangos, and M. Chergui

Citation: Review of Scientific Instruments 85, 103117 (2014); doi: 10.1063/1.4899062

View online: http://dx.doi.org/10.1063/1.4899062

View Table of Contents: http://scitation.aip.org/content/aip/journal/rsi/85/10?ver=pdfcov

Published by the AIP Publishing

\section{Articles you may be interested in}

Determining time resolution of microchannel plate detectors for electron time-of-flight spectrometers

Rev. Sci. Instrum. 81, 073112 (2010); 10.1063/1.3463690

Angle-resolving time-of-flight electron spectrometer for near-threshold precision measurements of differential cross sections of electron-impact excitation of atoms and molecules

Rev. Sci. Instrum. 79, 043105 (2008); 10.1063/1.2912824

Highly efficient time-of-flight spectrometer for studying low-energy secondary emission from dielectrics:

Secondary-electron emission from LiF film

Rev. Sci. Instrum. 74, 1274 (2003); 10.1063/1.1537044

High resolution pulsed field ionization photoelectron spectroscopy using multibunch synchrotron radiation: Timeof-flight selection scheme

Rev. Sci. Instrum. 70, 2615 (1999); 10.1063/1.1149818

High-resolution electron time-of-flight apparatus for the soft x-ray region

Rev. Sci. Instrum. 69, 3809 (1998); 10.1063/1.1149183
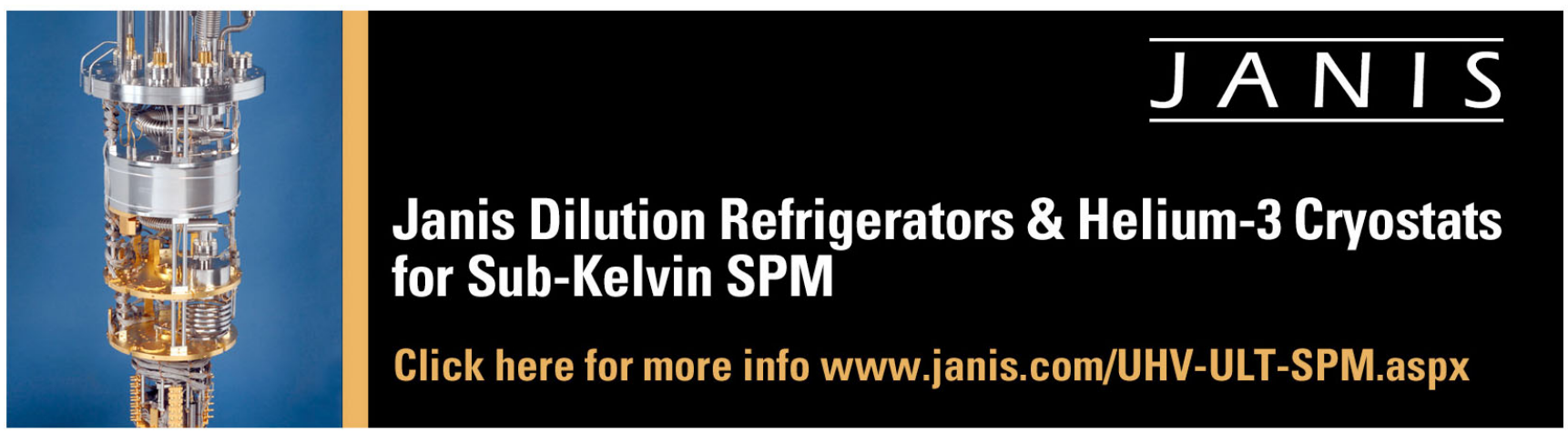


\title{
A simple electron time-of-flight spectrometer for ultrafast vacuum ultraviolet photoelectron spectroscopy of liquid solutions
}

\author{
C. A. Arrell, ${ }^{1}$, a) J. Ojeda, ${ }^{1}$ M. Sabbar, ${ }^{2}$ W. A. Okell, ${ }^{3}$ T. Witting, ${ }^{3}$ T. Siegel, ${ }^{3}$ Z. Diveki, ${ }^{3}$ \\ S. Hutchinson, ${ }^{3}$ L. Gallmann, ${ }^{2}$ U. Keller, ${ }^{2}$ F. van Mourik, ${ }^{1}$ R. T. Chapman, ${ }^{4}$ C. Cacho, ${ }^{4}$ \\ N. Rodrigues, ${ }^{4}$ I. C.E. Turcu, ${ }^{4}$ J. W.G. Tisch, ${ }^{3}$ E. Springate, ${ }^{4}$ J. P. Marangos, ${ }^{3}$ \\ and M. Chergui ${ }^{1}$ \\ ${ }^{1}$ Laboratory of Ultrafast Spectroscopy, ISIC, Station 6, Ecole Polytechnique Fédérale de Lausanne, CH-1015 \\ Lausanne, Switzerland \\ ${ }^{2}$ Physics Department, ETH Zurich, 8093 Zurich, Switzerland \\ ${ }^{3}$ Department of Physics, The Blackett Laboratory, Imperial College, London SW7 2AZ, United Kingdom \\ ${ }^{4}$ Central Laser Facility, STFC Rutherford Appleton Laboratory, Oxon OX11 0QX, United Kingdom
}

(Received 25 July 2014; accepted 10 October 2014; published online 28 October 2014)

\begin{abstract}
We present a simple electron time of flight spectrometer for time resolved photoelectron spectroscopy of liquid samples using a vacuum ultraviolet (VUV) source produced by high-harmonic generation. The field free spectrometer coupled with the time-preserving monochromator for the VUV at the Artemis facility of the Rutherford Appleton Laboratory achieves an energy resolution of $0.65 \mathrm{eV}$ at $40 \mathrm{eV}$ with a sub $100 \mathrm{fs}$ temporal resolution. A key feature of the design is a differentially pumped drift tube allowing a microliquid jet to be aligned and started at ambient atmosphere while preserving a pressure of $10^{-1}$ mbar at the micro channel plate detector. The pumping requirements for photoelectron (PE) spectroscopy in vacuum are presented, while the instrument performance is demonstrated with PE spectra of salt solutions in water. The capability of the instrument for time resolved measurements is demonstrated by observing the ultrafast (50 fs) vibrational excitation of water leading to temporary proton transfer. (C) 2014 AIP Publishing LLC. [http://dx.doi.org/10.1063/1.4899062]
\end{abstract}

\section{INTRODUCTION}

The development of time-resolved (picosecond to femtosecond) X-ray absorption spectroscopy (XAS) has allowed new insight into our understanding of photoinduced phenomena in molecular systems, biological systems, and materials. ${ }^{1,2}$ One of the main advantages of XAS is that it can deliver information about both the electronic structure (the density of unoccupied states) of the system under study, but also the geometric structure around a specific atom, via Xray near-edge absorption spectroscopy (XANES) or extended $\mathrm{X}$-ray absorption fine structure (EXAFS). It has recently been complemented by time-resolved X-ray emission spectroscopy $(\mathrm{XES})^{3,4}$ which probes the density of occupied states. However, both time resolved XAS and XES are typically implemented at large-scale installations, such as synchrotrons and X-ray free electron lasers (XFELs). In addition, these spectroscopic methods only provide information about the relative energies of the initial and final states, but not their absolute energy. Photoelectron spectroscopies (PES), in which an electron is detected rather than a photon, such as ultraviolet photoelectron spectroscopy (UPS) and X-ray photoelectron spectroscopy (XPS) are very well established techniques which directly provide the binding energy of a state. In addition it offers the advantage of a greater detection sensitivity than photon detection. PES is routinely used to probe the electronic structure of gas phase molecular systems and solids.

Photoelectron spectroscopy techniques require a high vacuum $\left(\sim 10^{-5}\right.$ mbar $)$ to allow photoelectron detection, and furthermore in the case of UPS a vacuum is also required for

a)Electronic mail: christopher.arrell@epfl.ch transmission of the probe light. XPS and UPS have recently been implemented in the static mode on high vapour pressure liquids thanks to the use of microjet technology that injects the liquid at high speed into the vacuum chamber in the form of a thin (typically a few tens of micrometers) jet. ${ }^{5}$ The extension of PES to the time domain has recently been achieved by Abel, Faubel, and co-workers ${ }^{6,7}$ who used ultrashort vacuum ultraviolet (VUV) pulses to probe the photoinduced dynamics of the pure water microjet or molecules dissolved in it. The source of ultrashort VUV photons was high harmonic generation (HHG).

In this article, we describe a simple differentially pumped electron time-of-flight (TOF) spectrometer for conducting ultrafast photoelectron spectroscopy of liquid samples probed with monochromatic VUV photons produced by HHG. The design is without additional electrostatic lenses or of a magnetic bottle design ${ }^{8}$ which can complicate operation when a liquid jet is being used.

The article is arranged as follows: Section II details the design principle of the instruments and describes the energy resolution and pumping requirements in more detail. Section III describes the time preserving monochromator at Artemis and the energy calibration. Section IV presents static measurements of potassium iodide in water and time-resolved proton transfer from vibrationally excited water. Conclusions and future outlooks are given in Sec. V.

\section{INSTRUMENT DESIGN}

The instrument was developed to investigate ultrafast excited state dynamics in molecular systems ${ }^{9,10}$ in solutions, for 
example a spin crossover (SCO) in metal ligand complexes such as iron(II)-tris-bipyridine $\left(\left[\mathrm{Fe}(\mathrm{bpy})_{3}\right]^{2+}\right) \cdot{ }^{11,12}$ These processes occur within $50 \mathrm{fs}^{4,13}$ and to observe both charge and structural dynamics during the spin crossover, element specificity is required. In the case of iron(II)-tris-bipyridine one could access the $\mathrm{N} 2 \mathrm{~s}(37.3 \mathrm{eV})$ and Fe $3 \mathrm{p}(52.7 \mathrm{eV})$ orbitals to gain information of the structural dynamics of the metal and ligand. High harmonic generation is a suitable source producing photons up to $150 \mathrm{eV}$ from a standard commercial high power laser. ${ }^{14}$

Conducting photoelectron spectroscopy from liquids ${ }^{5,15-18}$ is a tricky undertaking, principally as the liquid jet needs to be run in vacuum to allow for inelastic collection of the emitted photoelectrons and transmission of the VUV light. There are two troublesome obstacles to overcome in order to achieve this aim. First, the liquid jet has to be started at ambient atmosphere and run while the chambers are pumped down, otherwise the liquid jet nozzle would freeze. The second obstacle is the entrance to the drift tube has to be positioned less than $500 \mu \mathrm{m}$ away from the liquid target to avoid inelastic collisions of the collected electrons with the surrounding gaseous environment. Furthermore working with liquids in vacuum is an inherently awkward task, with a significant chance of jet misalignment causing significant ice build up and sample contamination of the spectrometer.

To overcome these issues the spectrometer design was simplified as much as possible to achieve the required energy resolution. Modelling of the energy resolution showed that a field free drift tube would suffice, and removed the complication of cleaning a retarding lens element following jet misalignment. To preserve the micro channel plate (MCP) detector below atmosphere conditions while the jet was aligned a molecular beam skimmer with an aperture of $100 \mu \mathrm{m}$ was used as the entrance of the spectrometer. The shape of the skimmer allows the liquid jet to be positioned less than $100 \mu \mathrm{m}$ from the skimmer without interfering with the jet collection or beam alignment. Furthermore the low leak rate of the skimmer allows the micro channel plates of the detector to remain at $1 \times 10^{-1}$ mbar (calibrated for gauge location) while the interaction chamber is at 1 bar. This significantly reduces the degassing time of the MCPs allowing electron detection within 30 min of jet alignment.

A detailed description of the energy resolution requirements, design, and pumping considerations follows.

\section{A. Energy resolution}

Given the temporal resolution required to resolve the spin crossover process the energy resolution of the TOF for this measurement is required to be in the range of $0.1-0.5 \mathrm{eV}$. Calculations of the ultimate energy resolution given by the data acquisition and geometric considerations for different drift tube lengths from 0.3 to $0.6 \mathrm{~m}$ were performed and are shown in Figure 1 which plots the resolution given by

$$
\Delta E=\sqrt{\left(\Delta E_{\mathrm{TDC}}\right)^{2}+\left(\Delta E_{\mathrm{MCP}}\right)^{2}}
$$

as a function of electron energy.

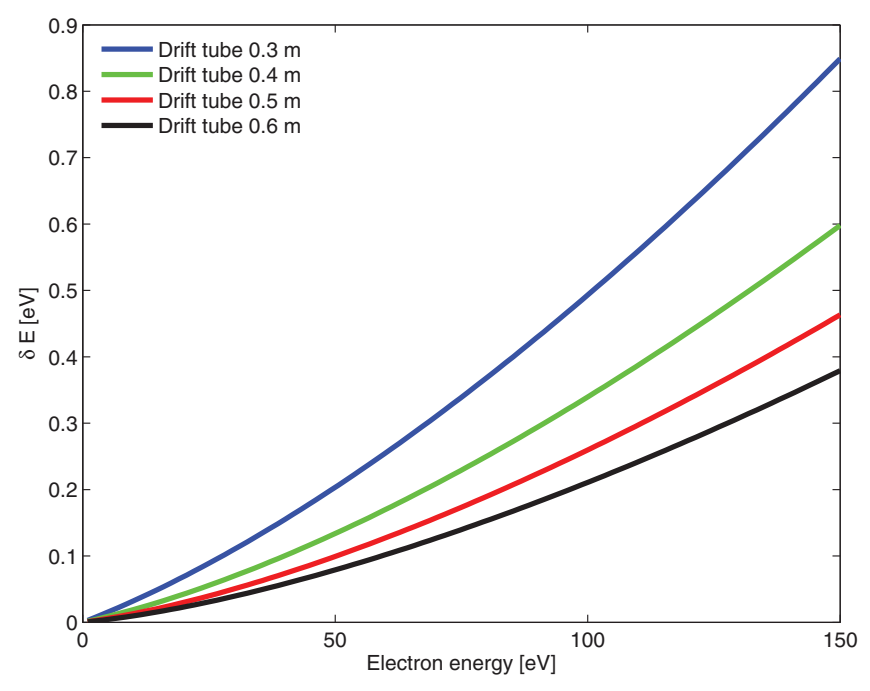

FIG. 1. Theoretical optimum energy resolution of a TOF with a $40 \mathrm{~mm}$ diameter MCP and a $100 \mathrm{ps}$ time bin in the data collection for different drift tube lengths.

The energy resolution limit originating from the time to digital convertor (TDC) time unit (100 ps) used for data collection is given by $\Delta E_{\mathrm{TDC}}$ and $\Delta E_{\mathrm{MCP}}$ is the limit of energy resolution originating from the two dimensional MCP array with no spatial resolution.

It is fair to assume the instrument's actual energy resolution will be twice the theoretical one accounting for stray magnetic fields and unavoidable inelastic scattering of the electrons. Mechanical requirements in the instrument design fixed the final drift tube length at $510 \mathrm{~mm}$ providing a theoretical energy resolution of $\Delta E=0.25 \mathrm{eV}$ at $100 \mathrm{eV}$.

\section{B. Design}

Given the drift tube length requirement for the energy resolution, the mechanical design of the instrument could be set and is shown in Figure 2. A copper entrance skimmer was sealed onto a $\mu$-metal drift tube, itself sealed onto a ConFlat (CF) DN200 $316 \mathrm{LN}$ flange sealing the detector from the interaction region. Behind this a CF DN150 cross was used to provide two DN150 pumping ports. The entire instrument was constructed from $316 \mathrm{LN}$ steel or copper through out and is shown in Figure 2.

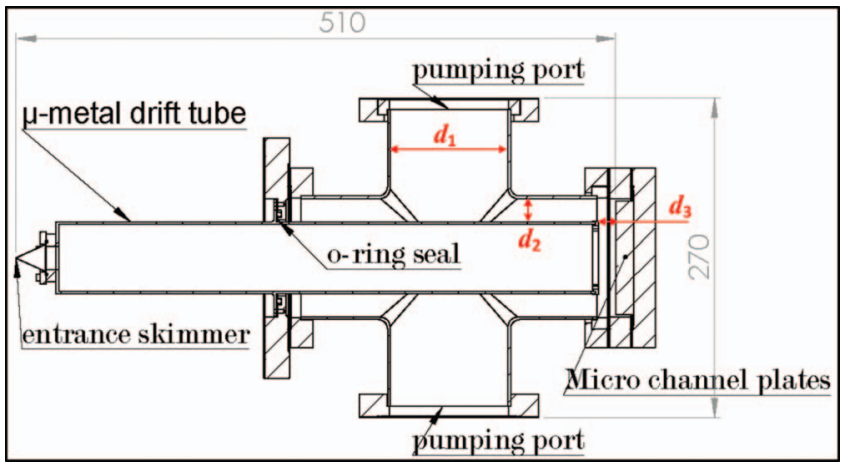

FIG. 2. Drawing of time-of-flight design. The characteristic dimensions (in $\mathrm{mm}$ ) of the design $-d_{1}, d_{2}$, and $d_{3}$ - were used in the conduction calculation and are referred to in the text. 
An important design criterion is the pumping conduction to the micro-channel plate where a tradeoff between the pumping conductance and the magnetic shielding is required. An entirely sealed $\mu$-metal drift tube would be best for an unperturbed electron flight path but it offers poor pumping conduction to the channel plates. The size of the gap was decided by calculating the net pumping speed of the spectrometer chamber.

Initially the gas flow regime present during the instrument operation can be verified by Knudsen number: ${ }^{19}$

$$
K_{n}=\frac{\eta}{P d} \sqrt{\frac{\pi}{2} \frac{R_{0} T}{M_{m}}},
$$

where $\eta$ is the coefficient of internal viscosity, $P$ is the gas pressure, $d$ is the characteristic dimension of the system, $R_{0}$ is the universal gas constant, $T$ is the temperature of the system, and $M_{m}$ is the gas molar mass.

For air at $20^{\circ} \mathrm{C}$, it can be approximated as ${ }^{19}$

$$
K_{n} \approx 0.066 / P d
$$

and

$$
K_{n} \approx 0.045 / P d
$$

for $\mathrm{H}_{2} \mathrm{O}$, where $P$ is in mbar and $d$ in mm. Molecular flow, i.e., when the mean free path of the molecules is greater than the characteristic dimension of the system, occurs from values of $K_{n}>0.5$, and viscous flow (where molecular collisions dominate) occurs for values of $K_{n}<0.01$

Under operating conditions, $K_{n}>>0.5$ and so the conduction of the system $(C)$ can be estimated using ${ }^{19}$

$$
C=\alpha A \sqrt{\frac{R_{0} T}{2 \pi M_{m}}},
$$

where $\alpha$ is the transmission probability and $A$ is the aperture area.

Conductance of a circular tube for air at $20{ }^{\circ} \mathrm{C}$ approximates to

$$
C=12 \frac{d^{3}}{z}\left[l s^{-1}\right]
$$

and

$$
C=15 \frac{d^{3}}{z}\left[l s^{-1}\right]
$$

for water where $d$ is the diameter and $z$ the length of the tube in $\mathrm{mm}$. For an aperture of area $A\left(\right.$ in $\left.\mathrm{cm}^{2}\right)$, the conductance is given by

$$
C=3.7\left(\frac{T}{M_{m}}\right)^{1 / 2} A\left[l s^{-1}\right] .
$$

The characteristic dimensions $\left(d_{1,2,3}\right)$ for the TOF design using a CF DN100 cross are shown in Figure 2, where the pumping system can be divided into the pumping port tube $\left(d_{1}\right)$, drift tube space $\left(d_{2}\right)$, and as an aperture in front of the MCP $\left(d_{3}\right)$. The latter is described as an aperture as the "length" of the tube is significantly shorter than the diameter. The pumping speed of the system is plotted in Figure 3, where the conduction is calculated as two tubes and an aperture in series,

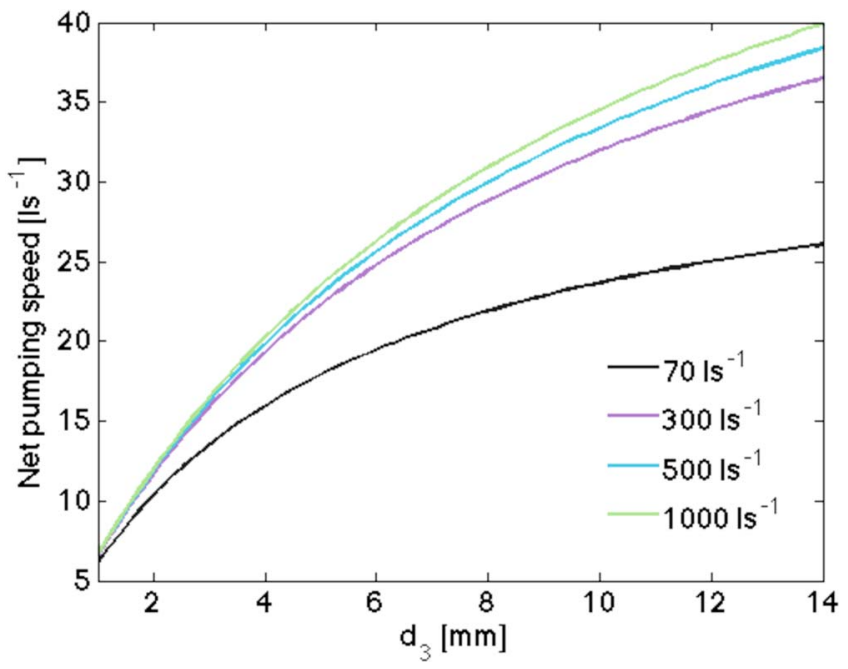

FIG. 3. Pumping speed of the TOF system for different sized turbo pumps as a function of $\mathrm{d}_{3}$, the distance between MCP and drift tube.

and the speed is

$$
\text { speed }=\left(\frac{1}{\text { Pump speed }}+\frac{1}{\text { Total conduction }}\right)^{-1},
$$

where

Total conduction $=\left(\frac{1}{2 \times \text { tube } 1}+\frac{1}{\text { tube } 2}+\frac{1}{\text { aperture }}\right)^{-1}$.

It can be seen from Figure 3 that there is little improvement in the pumping using a turbo pump larger than $3001 \mathrm{~s}^{-1}$, where as increasing the gap $\left(d_{3}\right)$ between the MCP front and drift tube end improves the pumping. Initially a spacing of 6 $\mathrm{mm}$ was chosen and $3001 \mathrm{~s}^{-1}$ turbo pumps giving a net pumping of $\sim 25 \mathrm{l} \mathrm{s}^{-1}$ at the MCP plates. The TOF design allows the gap $\left(d_{3}\right)$ to be increased if required by changing the spacer flange before the MCP assembly.

\section{Pumping}

The pressure localized around the jet and the background pressure in the chamber are both of importance for VUV measurements. Reducing the localized pressure of the liquid jet is required to increase the inelastic mean free path of the emitted photoelectrons, and to avoid absorption of the VUV probe photons. Furthermore the gas load in the spectrometer chamber needs to be controlled to ensure a suitable operating pressure for the micro channel plates.

A multi-pronged approach was taken to achieve operational pressures. First the evaporation load from the jet was reduced by cooling the injected liquid into the chamber. The experimental and spectrometer chamber pressures against jet temperature are shown in the inset of Figure 4. Cooling the jet to just above freezing point reduced the background load by a factor of 2 . Further reduction in the evaporation contribution was achieved by using a new approach for collecting the liquid jet. In previous liquid jet studies the jet has simply been frozen within the experimental chamber on a suitable cold finger. ${ }^{17}$ In the current work, a further skimmer was used 


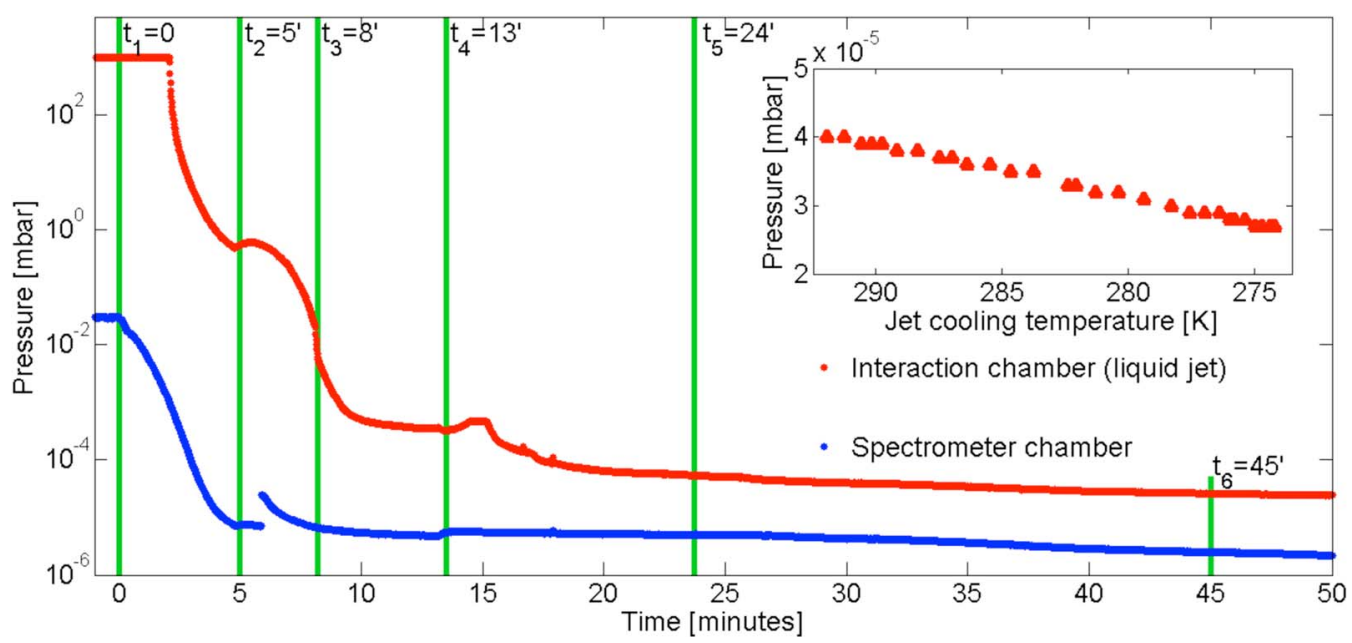

FIG. 4. Main plot: pump down progress of interaction and spectrometer chamber with the liquid jet in operation. Inset: pressure decrease in the interaction chamber as the liquid jet is cooled.

to collect the liquid jet just below the interaction region and exited into a further differentially pumped collection bottle below the experimental chamber. In this way the jet surface area exposed to the experimental chamber vacuum was limited to $\sim 0.05 \mathrm{~mm}^{2}$ as opposed to $\sim 10 \mathrm{~mm}^{2}$ in previous setups. The bottle was maintained at $1{ }^{\circ} \mathrm{C}$ and $\sim 7$ mbar. Care has to be taken to avoid lower pressures in the bottle as the static water would freeze. This sample collection method also opens the possibility of sample circulation for reuse. To avoid gradual ice formation blocking the collection skimmer, conductive non magnetic heating was used.

The pumping of the experimental and spectrometer chambers requires four pumping systems, with the pumpdown pressures shown in Figure 4. Initially with the spectrometer chamber pumped with two turbo molecular pumps (Agilent TwisTorr 304FS), a scroll pump (Edwards nXDS10i) and control valve were used to pump the experimental chamber to 1 mbar $\left(t_{1}-t_{2}\right)$. Careful control of this stage is required as large jet walk-off occurs in the fluid flow regime further exacerbated by mechanical walk-off from the manipulator bellows during pumping. On entering the molecular flow regime at $\mathrm{t}_{2}$ a $1000 \mathrm{l} \mathrm{s}^{-1}$ turbo molecular pump (Agilent Turbo V-1001) reduces the pressure to $8 \times 10^{-3} \mathrm{mbar}$ whereupon the pumping efficiency of water vapour is negligible. At this point $\left(t_{3}\right)$ a cold finger immediately behind the turbo pump exhaust is filled. Within 5 min a further two, 21 cold fingers in the experimental chamber are filled providing $\sim 8000 \mathrm{~s}^{-1}$ of pumping for water vapour, rapidly achieving a stable operating pressure of $5 \times 10^{-5}$ mbar. After $20 \mathrm{~min}$ or so the cooling of the liquid jet is turned on, reducing further the chamber pressure to a stable $2 \times 10^{-5}$ mbar in the experimental chamber and $2 \times 10^{-6}$ mbar in the spectrometer chamber. The pressures of the drift tube quoted here include a calibration factor of 1.8 between the MCP position and the pressure gauge location. This was measured by placing a further gauge in place of the MCP.

\section{EXPERIMENTAL SETUP}

The instrument was installed at the Artemis beamline of the Central Laser Facility in the UK. ${ }^{20-22}$

The fundamental laser, a multi-pass titanium-sapphire amplifier, produced up to $10 \mathrm{~mJ}, 800 \mathrm{~nm}$ pulses with a FWHM of $30 \mathrm{fs}$ at $1 \mathrm{kHz}$. For time resolved measurements the laser output was split 20:80 for probe:pump (Fig. 5).

The probe beam was focused into a highly localized target of atomic argon where HHG produced a plateau of harmonics, with a $50 \mathrm{eV}$ cut-off energy. After generation, the high harmonics were monochromated (see Sec. III A) to give time-preserved, monochromatic VUV pulses. After monochromation, the VUV pulses were focused to a $50 \mu \mathrm{m}$ FWHM diameter spot size with a gold coated toroidal

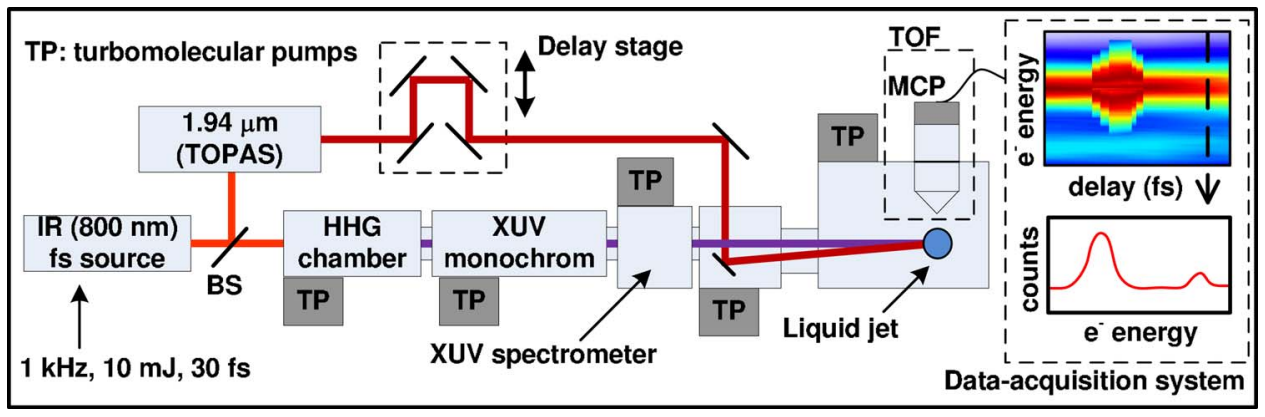

FIG. 5. Schematic of time resolved photoelectron spectroscopy setup. 


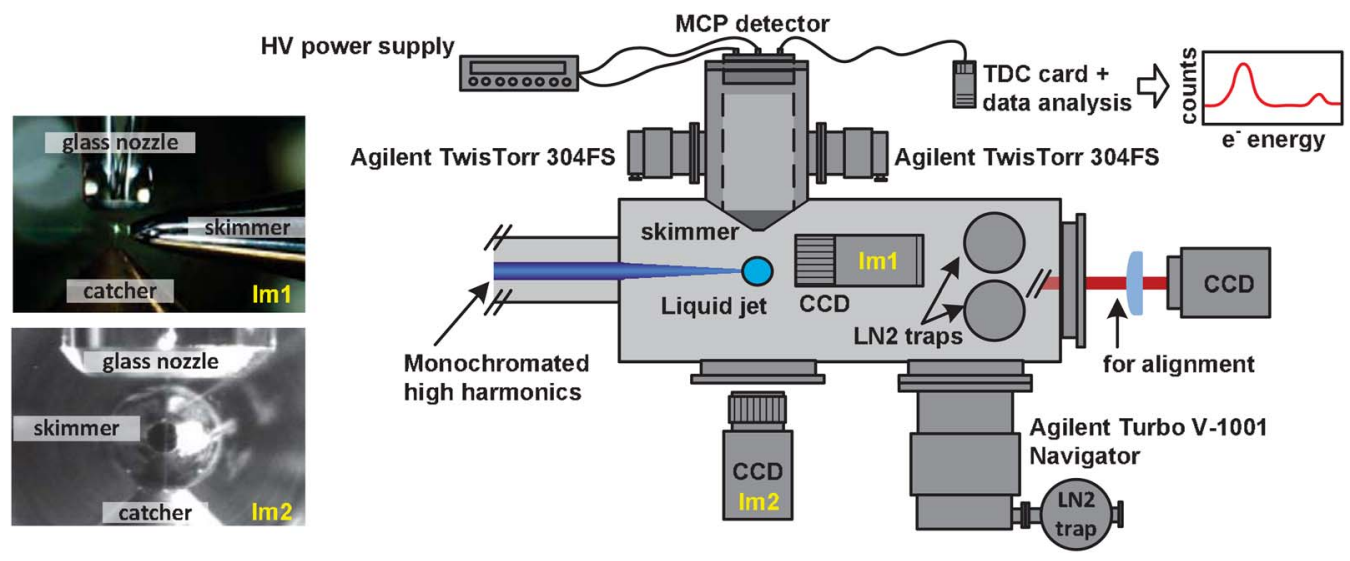

FIG. 6. Inset: the liquid jet in operation. The entrance skimmer to the TOF is on the right, and the catcher skimmer is below. The jet diameter is $30 \mu \mathrm{m}$. Main figure: schematic of the experimental chamber setup with time of flight.

mirror at grazing incidence. The monochromatic photon flux was $\approx 1 \times 10^{9}$ photons/s, and about 10 electrons/s were detected on the MCP. The remaining $80 \%$ beam energy was fed to an optical parametric amplifier (OPA, TOPAS Light Conversion) to generate about $100 \mu \mathrm{J}$ at $1.94 \mu \mathrm{m}\left(5000 \mathrm{~cm}^{-1}\right)$, to excite the combination (stretch, bend) mode of the water molecule. Time-resolved photoelectron spectra were observed as a function of the delay between pump and probe pulses.

\section{A. Monochromation and calibration}

The time preserving monochromator at Artemis has four gratings matched to either high energy resolution ( $\Delta E \sim 0.3 \mathrm{eV}$ and $50 \mathrm{fs}$ ) or high temporal resolution (20 fs and $\Delta E=1.7 \mathrm{eV}$ ). The temporal stretch of the VUV pulse by the monochromator is limited by using the gratings with the grooves parallel to the beam direction. By carefully collimating the VUV beam before the grating, the required number of grooves are illuminated for a given energy resolution. A full description of the monochromator is given elsewhere. ${ }^{22}$ The measurements presented here used a grating with groove density of $500 \mathrm{gr} / \mathrm{mm}$ and a theoretical energy resolution of $\Delta E$ $=1.0 \mathrm{eV}$ at $62 \mathrm{eV}$.

Calibration of the instrument was performed by measuring the first ionization energy of neon placing an effusive gas target in place of the liquid jet, the position of which was referenced by a CCD mounted inside the chamber (labelled Im1 in Figure 6). The angle of the grating in the monochromator was rotated to select individual harmonics from the 21 st to the 29th, with a photoelectron spectrum collected at each angle. The spacing of photoelectron kinetic energies of the different harmonics is $2 \omega$ of the fundamental laser wavelength (Figure 7). The fitting of this parameter to collected spectra allowed the effective drift tube length to be calculated - the distance between the photoelectron source and the MCPs. The error in the measured binding energy position compared to $2 \omega$ was less than $0.1 \mathrm{eV}$ over $30 \mathrm{eV}$. An example of a monochromated harmonic is shown in Figure 8, with a FWHM of $0.65 \mathrm{eV}$.

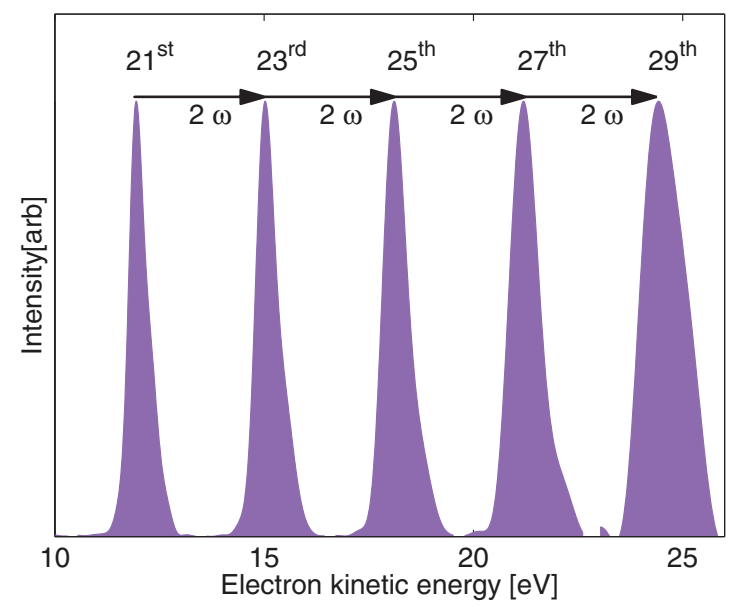

FIG. 7. Photoelectron spectra of the first ionization potential of neon measured with harmonics 21-29. Fitting the harmonic spacing to $2 \omega$ of the fundamental gave an effective drift tube length of $0.54 \mathrm{~m}$.

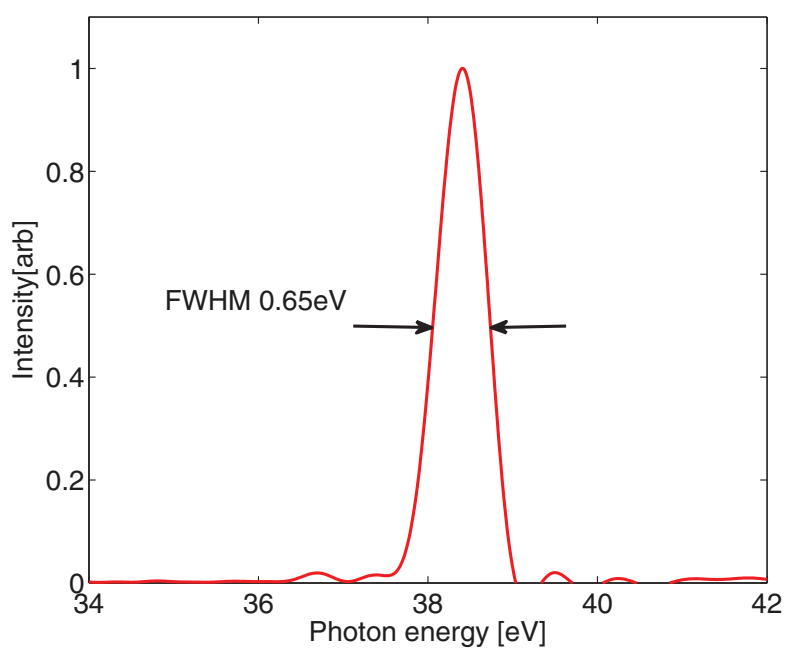

FIG. 8. Photoelectron spectrum of the 25th harmonic of the fundamental laser. 


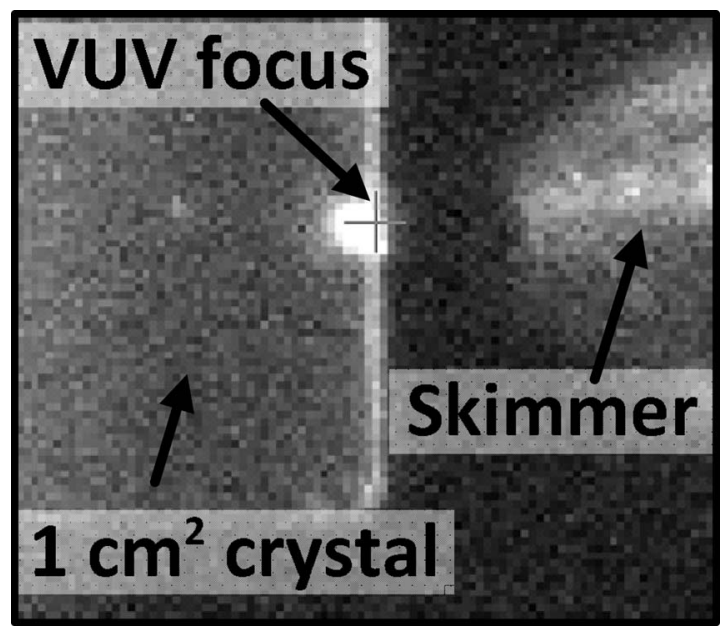

FIG. 9. Referencing the VUV focus using a scintillator crystal.

\section{B. Alignment}

Alignment of the liquid jet, optical beams, and the electron spectrometer input is a critical issue for PES of liquids. The dimensions of the VUV focal spot $(\sim 80 \mu \mathrm{m})$ and the jet's diameter $(25 \mu \mathrm{m})$ make misalignments of a few micrometers significant.

Alignment is complicated by the difficulty of visualising the VUV - required to achieve spatial overlap for pumpprobe measurements. A $1 \mathrm{~cm}^{2}, 1 \mathrm{~mm}$ thick scintillator crystal (LuAG:Ce from Crytur) itself imaged by a photo multiplier camera (Andor Luca R) was used, allowing the scintillation from the VUV to be seen. This method (Figure 9) enabled the VUV to be visually spatially overlapped with the pump pulse.

\section{RESULTS AND ANALYSIS}

Using the monochromatic $38.4 \mathrm{eV}$ VUV probe, photoelectron measurements were taken of a $2 \mathrm{M}$ concentration solution of potassium iodide (KI) in water. For these measurements the jet was placed $300 \mu \mathrm{m}$ from the entrance skimmer (referenced by the internal CCD). The external jacket of the liquid jet was cooled to $1^{\circ} \mathrm{C}$ and the jet flow rate was set to

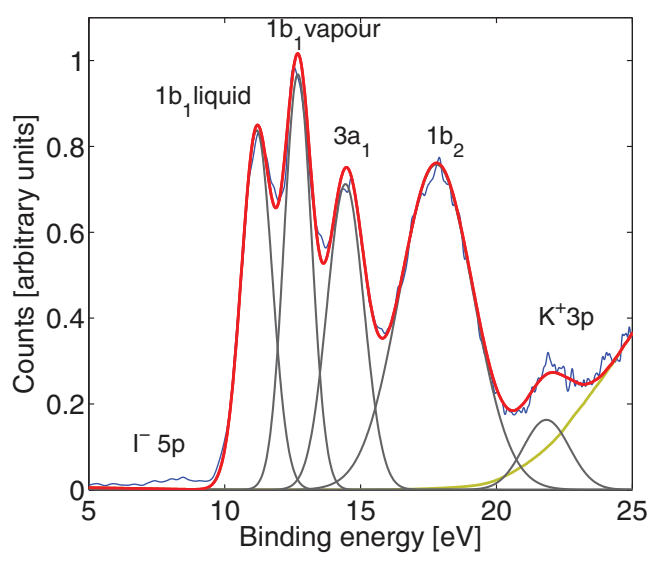

FIG. 10. Binding energies of the first three water valence orbitals and $\mathrm{K}^{+} 3 \mathrm{p}$ and $\mathrm{I}^{-} 5 \mathrm{p}$. Blue curve raw data, red curve Fourier filtered data, black curve Gaussian fit to orbitals, green curve secondary electron fit.
TABLE I. Measured binding energies for the first three water orbital, $\mathrm{K}^{+} 3 \mathrm{p}$ and $\mathrm{I}^{-} 5 \mathrm{p}$.

\begin{tabular}{lcc}
\hline \hline Orbital & Binding energy [eV] & FWHM [eV] \\
\hline $\mathrm{I}^{-} 5 \mathrm{p}$ & 8 & $\sim$ \\
$1 \mathrm{~b}_{1}$ liquid & 11.2 & 1.3 \\
$1 \mathrm{~b}_{1}$ gas & 12.7 & 1.2 \\
$3 \mathrm{a}_{1}$ & 14.4 & 1.6 \\
$1 \mathrm{~b}_{2}$ & 17.8 & 3.2 \\
$\mathrm{~K}^{+} 3 \mathrm{p}$ & 21.8 & 2.0 \\
\hline \hline
\end{tabular}

$0.5 \mathrm{~m} \mathrm{l} \mathrm{s}^{-1}$, producing a liquid flow velocity of $\sim 10 \mathrm{~m} \mathrm{~s}^{-1}$. By using a mechanical switch for the liquid pump, samples could be changed without moving the jet position. A coloured dye was used to measure the sample exchange rate time and measured as 20-30 min was used as a benchmark for full sample exchange at the target area.

In line with previous measurements ${ }^{23-25}$ it was found that charge carriers were needed to measure the photoelectron spectrum of a water sample. The high resistivity $(18 \mathrm{M} \Omega)$ of the high performance liquid chromatography (HPLC) grade water used produced a large charging of the sample by the motion over the polyether ether ketone (PEEK) liquid jet tubing and fused silica nozzle. Test measurements found the static charging of the jet was neutralized by the addition of at least $5 \mathrm{mM}$ of $\mathrm{NaCl}$.

The PE spectrum of a $2 \mathrm{M}$ solution of $\mathrm{KI}$ in water was measured (Figure 10). Identifiable are the first three molecular orbitals of water $\left(1 b_{1}, 3 a_{1}\right.$, and the $\left.1 b_{2}\right)$ and the $K^{+} 3 p$ and $\mathrm{I}^{-} 5 \mathrm{p}$ binding energies of the cation and anions. Gaussians were fitted to the Fourier filtered spectrum with the secondary electron contribution removed. The fitted binding energy and peak FWHM are shown in Table I. Demonstrated by the figure is the ability to measure both the element specific binding energies of the anion and cation and also the molecular orbitals of water.

The $1.5 \mathrm{eV}$ splitting in the $1 \mathrm{~b}_{1}$ HOMO peak (Figure 11) originates from the presence of hydrogen bonds in liquid

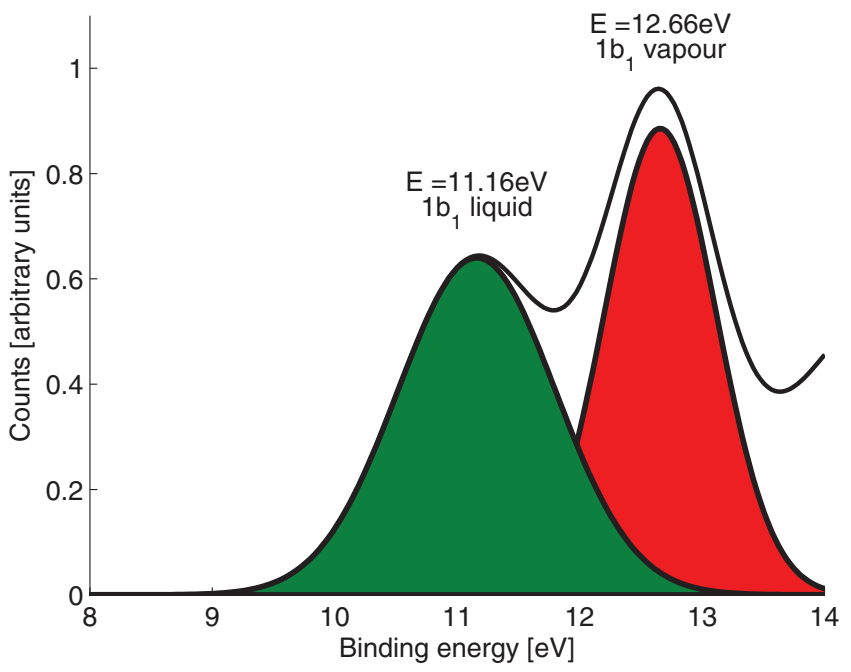

FIG. 11. Splitting of the HOMO $\left(1 b_{1}\right)$ of water in gas and liquid phase samples. 


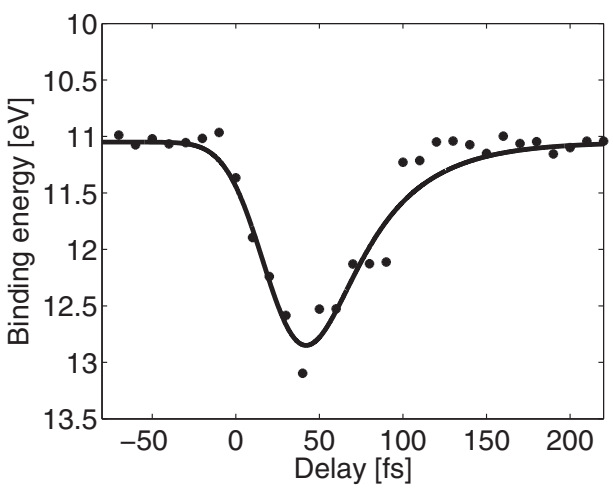

FIG. 12. Ultra-fast binding energy shake-up of the centre of mass of liquid water HOMO following vibrational excitation of the combination stretchbend mode.

water. The $\mathrm{H}$-bond is formed between the $\delta+\mathrm{H}$ atom and the $\delta$-lone pair $\left(2 \mathrm{p}_{x}\right)$ of electrons on the oxygen. ${ }^{26}$ It is these electrons which are probed by measuring the $1 \mathrm{~b}_{1}$ binding energy, a molecular orbital which is entirely located on the oxygen. The clear and distinct splitting in binding energy between the liquid and gas phase (Figure 11) provides a direct handle into the dynamics of the intermolecular bond by measuring an intramolecular orbital. It is hard to remove the gas phase contribution surrounding the jet as the monochromator is based upon a 1:1 imaging arrangement, where ultimately the high harmonic generation interaction volume is re-imaged at the toroidal mirror focus. It was estimated that for phase matched conditions the high harmonic interaction volume had a diameter of 50-80 $\mu \mathrm{m}-4$ times the liquid jet diameter. Previously the gas phase contribution has been removed by aligning the jet to obtain a pure gas phase spectrum and then subtracting this from the combined gas and liquid phase spectrum. In the presented measurements however, the large splitting in the $1 b_{1}$ HOMO gives a clear difference in the liquid and gas phase contributions.

The ability of the instrument to resolve rapid charge transfer in liquid phase was demonstrated with a pump probe measurement in which the water sample was vibrationally excited. The output of the multi pass amplifier was split with $2 \mathrm{~mJ}$ pumping an OPA to produce $1.95 \mu \mathrm{m}$ pulses. This optical pump focus was spatially and temporally overlapped at the liquid jet with the VUV probe in front of the time-of-flight spectrometer. The wavelength of the IR pump excited the combination stretch-bend vibrational mode of the water molecules.

What is seen in Figure 12 is the binding energy shake-up observed after vibrational excitation. This short lived shakeup is a likely effect of the close intermolecular distances between neighbouring oxygens in liquid water and will be discussed in more detail elsewhere. ${ }^{27}$

\section{v. CONCLUSION}

We have demonstrated a simple electron TOF design to conduct ultrafast photoelectron spectroscopy of liquid solutions. Element specificity, identification of molecular orbitals and sensitivity to intermolecular bonds has been shown. Fur- thermore the temporal dynamics of the binding energy shakeup following vibrational excitation of water has been followed for the first time with less than 100 fs temporal resolution.

This novel spectrometer design now opens the gateway to understanding photo-induced processes in molecular systems in a lab based environment with the unprecedented temporal resolution provided by HHG.

\section{ACKNOWLEDGMENTS}

Funding from NCCR MUST, R'EQUIP proposal number 206021_145057 of the Swiss NSF, EPSRC (UK) Grant No. and ERC ASTEX project 290467 are acknowledged. The research leading to these results has received funding from LASERLAB-EUROPE (Grant Agreement No. 284464, EC's Seventh Framework Programme).

${ }^{1}$ T. J. Penfold, C. J. Milne, and M. Chergui, "Recent advances in ultrafast X-ray absorption spectroscopy of solutions," in Advances in Chemical Physics, edited by S. A. Rice, and A. R. Dinner (John Wiley \& Sons, Inc., 2013), Vol. 153, pp. 1-41.

${ }^{2}$ C. J. Milne, T. J. Penfold, and M. Chergui, "Recent experimental and theoretical developments in time-resolved x-ray spectroscopies," Coordination Chemistry Reviews 277-278, 44-68 (2014).

${ }^{3}$ G. Vankó, P. Glatzel, V.-T. Pham, R. Abela, D. Grolimund, C. Borca, S. Johnson, C. Milne, and C. Bressler, "Picosecond time-resolved X-ray emission spectroscopy: Ultrafast spin-state determination in an iron complex," Angew. Chem. 122, 6046-6048 (2010).

${ }^{4}$ W. Zhang, R. Alonso-Mori, U. Bergmann, C. Bressler, M. Chollet, A. Galler, W. Gawelda, R. G. Hadt, R. W. Hartsock, T. Kroll, K. S. Kjær, K. Kubiček, H. T. Lemke, H. W. Liang, D. A. Meyer, M. M. Nielsen, C. Purser, J. S. Robinson, E. I. Solomon, Z. Sun, D. Sokaras, T. B. van Driel, G. Vankó, T.-C. Weng, D. Zhu, and K. J. Gaffney, "Tracking excited-state charge and spin dynamics in iron coordination complexes," Nature (London) 509, 345-348 (2014).

${ }^{5}$ B. Winter and M. Faubel, "Photoemission from liquid aqueous solutions," Chem. Rev. 106, 1176-1211 (2006).

${ }^{6}$ O. Link, E. Lugovoy, K. Siefermann, Y. Liu, M. Faubel, and B. Abel, "Ultrafast electronic spectroscopy for chemical analysis near liquid water interfaces: Concepts and applications," Appl. Phys. A 96, 117-135 (2009).

${ }^{7}$ M. Faubel, K. R. Siefermann, Y. Liu, and B. Abel, "Ultrafast soft X-ray photoelectron spectroscopy at liquid water microjets," Acc. Chem. Res. 45, 120-130 (2012).

${ }^{8}$ A. Kothe, J. Metje, M. Wilke, A. Moguilevski, N. Engel, R. Al-Obaidi, C. Richter, R. Golnak, I. Y. Kiyan, and E. F. Aziz, "Time-of-flight electron spectrometer for a broad range of kinetic energies," Rev. Sci. Instrum. 84, 023106 (2013).

${ }^{9} \mathrm{C}$. Bressler and M. Chergui, "Molecular structural dynamics probed by ultrafast x-ray absorption spectroscopy," Annu. Rev. Phys. Chem. 61, 263282 (2010).

${ }^{10}$ A. Lübcke, F. Buchner, N. Heine, I. V. Hertel, and T. Schultz, "Timeresolved photoelectron spectroscopy of solvated electrons in aqueous nai solution," Phys. Chem. Chem. Phys. 12, 14629-14634 (2010).

${ }^{11}$ A. Cannizzo, C. Milne, C. Consani, W. Gawelda, C. Bressler, F. Van Mourik, and M. Chergui, "Light-induced spin crossover in fe (ii)-based complexes: The full photocycle unraveled by ultrafast optical and x-ray spectroscopies," Coord. Chem. Rev. 254, 2677-2686 (2010).

${ }^{12}$ W. Gawelda, A. Cannizzo, V.-T. Pham, A. El Nahhas, C. J. Milne, R. van der Veen, C. Bressler, and M. Chergui, "Light-induced spin crossover probed by ultrafast optical and x-ray spectroscopies," CHIMIA Int. J. Chem. 61, 179-183 (2007).

${ }^{13}$ C. Bressler, C. Milne, V. Pham, A. ElNahhas, R. M. van der Veen, W. Gawelda, S. Johnson, P. Beaud, D. Grolimund, M. Kaiser, C. N. Borca, G. Ingold, R. Abela, and M. Chergui, "Femtosecond XANES study of the Light-Induced spin crossover dynamics in an Iron(II) complex," Science 323, 489-492 (2009).

${ }^{14}$ J. Tisch, R. Smith, M. Ciarrocca, J. Muffett, J. Marangos, and M. Hutchinson, "Angularly-resolved high-order harmonic generation in helium," J. Mod. Opt. 41, 1163-1172 (1994). 
${ }^{15}$ M. Faubel, S. Schlemmer, and J. Toennies, "A molecular beam study of the evaporation of water from a liquid jet," Z. Phys. D. 10, 269-277 (1988).

${ }^{16}$ B. Winter, "Liquid microjet for photoelectron spectroscopy," Nucl. Instrum. Methods Phys. Res., Sect. A 601, 139-150 (2009).

${ }^{17}$ R. Seidel, S. Thürmer, and B. Winter, "Photoelectron spectroscopy meets aqueous solution: Studies from a vacuum liquid microjet," J. Phys. Chem. Lett. 2, 633-641 (2011).

${ }^{18}$ A. Lübcke, F. Buchner, N. Heine, I. V. Hertel, and T. Schultz, "Timeresolved photoelectron spectroscopy of solvated electrons in aqueous $\mathrm{NaI}$ solution," Phys. Chem. Chem. Phys. 12, 14629-14634 (2010).

${ }^{19}$ J. M. Lafferty, Foundations of Vacuum Science and Technology (Wiley, New York, 1998).

${ }^{20}$ I. E. Turcu, E. Springate, C. A. Froud, C. M. Cacho, J. L. Collier, W. A. Bryan, G. J. Nemeth, J. P. Marangos, J. W. Tisch, R. Torres et al., "Ultrafast science and development at the artemis facility," $R O$ MOPTO 2009 (International Society for Optics and Photonics, 2009), pp. 746902-746902.

${ }^{21}$ C. Froud, S. Bonora, E. Springate, A. Langley, D. Wolff, S. Blake, P. Brummitt, A. Cavalleri, S. Dhesi, L. Poletto et al., "Artemis: A sub 10-fs xuv source for ultrafast time-resolved science," Rutherford Appleton Laboratory, Central Laser Facility Annual Report 2007, 2007 (2006).

${ }^{22}$ F. Frassetto, C. Cacho, C. A. Froud, I. E. Turcu, P. Villoresi, W. A. Bryan, E. Springate, and L. Poletto, "Single-grating monochromator for extreme-ultraviolet ultrashort pulses," Opt. Express 19, 19169-19181 (2011).

${ }^{23}$ Y. Tang, Y.-I. Suzuki, H. Shen, K. Sekiguchi, N. Kurahashi, K. Nishizawa, P. Zuo, and T. Suzuki, "Time-resolved photoelectron spectroscopy of bulk liquids at ultra-low kinetic energy," Chem. Phys. Lett. 494, 111-116 (2010).

${ }^{24}$ A. T. Shreve, T. A. Yen, and D. M. Neumark, "Photoelectron spectroscopy of hydrated electrons," Chem. Phys. Lett. 493, 216-219 (2010).

${ }^{25}$ N. Preissler, F. Buchner, T. Schultz, and A. Lübcke, "Electrokinetic charging and evidence for charge evaporation in liquid microjets of aqueous salt solution," J. Phys. Chem. B 117, 2422-2428 (2013).

${ }^{26}$ I. D. Brown, "On the geometry of O-H...O hydrogen bonds," Acta Crystallogr. Sec. A 32, 24-31 (1976).

${ }^{27}$ C. A. Arrell, "Frustrated proton transfer in IR-excited liquid water probed by ultrafast photoelectron spectroscopy," (unpublished). 